\title{
Differential and decreased expression of Fibulin 1, Fibulin 2, and Fibulin 5 in metastases to disparate organ sites in breast cancer.
}

Shahan Mamoor ${ }^{1}$

1 Thomas Jefferson School of Law

San Diego, CA 92901

mamoorsk@tjsl.edu

Breast cancer is a leading killer of women in the United States (1) and the main reason women diagnosed with breast cancer die is metastasis, or spread of the cancer from the breast to a distant organ site $(2,3)$. We mined public datasets $(4,5)$ to perform systems-level analyses of the most significant differences in gene expression (6) between breast cancers in humans and the metastases they generate. We found that fibulin-1, fibulin-2 and fibulin-5 were among the genes whose expression was most different between primary tumors of the breast and metastases to both the brain and the soft tissues. Moreover, we found uniform and significantly decreased expression of each differentially expressed fibulin gene in both tissue types. Fibulins-1, -2 and -5 should be targeted to assess whether over-expression of these genes can halt, delay or reverse metastasis in women with breast cancer.

Keywords: breast cancer, metastasis, brain metastasis, soft tissue metastasis, tumor transcriptome analysis, systems oncology, targeted therapeutics in breast cancer. 


\section{Introduction}

There are over a quarter of a million cases of breast cancer in the United States each year and breast cancer is only second to lung cancer in terms of cancer prevalence in women (1). Over 40,000 women expire after being diagnosed with breast cancer (1) and the major reason for these deaths is metastasis, or spread of the cancer from the primary site, the breast, to a different organ site, such as the liver, lungs, brain, soft tissues, or bone $(2,3)$. There are no therapeutics strategies available that specifically target the metastatic process in breast cancer. In this study we compared the transcriptomes of primary tumors of the breast with that of metastases to the brain (4) and to the soft tissues (5) using two independently generated public datasets to identify in an unbiased and systems-level fashion the genes whose expression was most different between primary breast cancers and the metastases they generate. We found that the fibulin genes fibulin-1, fibulin-2, and fibulin-5 were among the most differentially expressed genes in metastases to the brain and to the soft tissues. Fibulin genes were expressed at significantly lower levels in metastatic tissues from women with breast cancer and xenograft models should be employed to assess whether overexpression of fibulins can serve as a novel therapeutic strategy to halt, delay or reverse the metastatic process in breast cancer.

\section{$\underline{\text { Methods }}$}

GSE125989 (4) and GSE124648 (5) were utilized for this analysis in conjunction with GEO2R. Investigators that created GSE125989 used Affymetrix Human Genome U133A 2.0 microarray technology to generate transcriptome data. In GSE125989, $n=16$ 
brain metastasis tissue samples and $n=16$ primary breast tumor samples. These samples were matched (from the same 16 patients). Investigators that created GSE124648 used Affymetrix Human Genome U133A microarray technology to generate transcriptome data. In GSE124648, $n=147$ primary breast tumors from women diagnosed with hormone-receptor positive breast cancer, including HER2+ and HER2patients, including ER+ patients, from stage IA to stage IIIC, obtained by fine needle aspiration or by surgery) and $n=32$ soft tissue metastases. In this study, the Benjamin and Hochberg method of $p$-value adjustment was utilized, and log-transformation of data was set to "Auto-detect". The raw p-value is utilized here. The NCBI generated category of platform annotation was utilized. A statistical test was performed to evaluate the significance of difference in RNA expression values between each differentially expressed gene in primary breast tumors and brain metastases or soft tissue metastases by a two-tailed, unpaired t-test with Welch's correction using PRISM (8.1.2) (227). Regardless of whether differences in exact mRNA expression levels are considered statistically significant, all differentially expressed genes described here are determined to be statistically significant with respect to global differential expression.

\section{$\underline{\text { Results }}$}

We compared the transcriptomes of tumors isolated from the breasts of women diagnosed with breast cancer, with the transcriptomes of metastatic tissues from women diagnosed with breast cancer, from both the brain and the soft tissues, by global differential gene expression analysis using two independent datasets $(4,5)$. We found that across datasets, fibulins 1,2 , and 5 were among the most differentially expressed 
genes in the breast cancer metastasis transcriptome in both the brain and the soft tissues.

Fibulins 1,2 and 5 are differentially expressed in metastases to the brain and the soft tissues in patients with breast cancer.

We identified fibulin-1, fibulin-2 and fibulin-5 as differentially expressed in metastases to the brain and to the soft tissues when compared to primary tissues of the breast. In the brain, the differential expression of Fibulin-1 was ranked 122 out of 22277 total transcripts detected and measured, while in the soft tissues the differential expression of Fibulin-1 was ranked 65 out of 22283 total transcripts detected and measured. The differential expression of Fibulin-2 was ranked 165 out of 22277 total transcripts detected and measured in the brain, and ranked 56 with respect to differential expression in soft tissues out of 22283 total transcripts detected and measured. In the brain, the differential expression of Fibulin-5 was ranked 129, while in the soft tissues the differential expression of Fibulin-1 was ranked 63. The differential expression of each these genes relative to the total transcriptome was statistically significant (Table 1; Fibulin-1, $p=3.27 \mathrm{E}-04$ in the brain and $\mathrm{p}=1.44 \mathrm{E}-21$ in soft tissues; Fibulin-2, $p=6.92 E-04$ in the brain and $p=5.22 E-22$ in soft tissues; Fibulin-5, $p=3.67 E-04$ in the brain and $p=1.33 E-21$ in soft tissues).

Fibulin-1 is expressed at significantly lower levels in metastases to the brain and the soft tissues when compared to primary tumors of the breast.

We extracted the exact mRNA expression values for fibulin-1 from each primary tumor sample and from each metastasis sample, in both the brain and the soft tissues. Fibulin-1 was expressed at significantly lower levels in both the brain and the soft 
tissues when compared to primary tumors of the breast (Figure 1; $p=0.0004$ for the brain and $p<0.0001$ for the soft tissues).

Fibulin-2 is expressed at significantly lower levels in metastases to the brain and the soft tissues when compared to primary tumors of the breast.

Next, we extracted the exact mRNA expression values for fibulin-2 from each primary tumor sample and from each metastasis sample, in both the brain and the soft tissues. Fibulin-2 was also expressed at significantly lower levels in both the brain and the soft tissues when compared to primary tumors of the breast (Figure 2; $p=0.0003$ for the brain and $p<0.0001$ for the soft tissues).

Fibulin- 5 is expressed at significantly lower levels in metastases to the brain and the soft tissues when compared to primary tumors of the breast.

Finally, we extracted the exact mRNA expression values for fibulin-5 from each primary tumor sample and from each metastasis sample, in both the brain and the soft tissues. Fibulin-5 was also expressed at significantly lower levels in both the brain and the soft tissues when compared to primary tumors of the breast (Figure 3; $p=0.0008$ for the brain and $p<0.0001$ for the soft tissues).

\section{Discussion}

Breast cancer is a serious medical problem in the United States with over 250,000 women diagnosed with the disease each year and over 40,000 deaths from the cancer (1). Metastasis to distant organ sites is the reason humans die from cancer (2, 3). We focused on the metastatic process in breast cancer to try and understand the genes whose expression changed the most between primary tumors of the breast and metastatic tissues from women with breast cancer (6). We analyzed transcriptome data 
from two metastatic sites, the brain and the soft tissues, using independent datasets (4, 5). Usage of separate datasets was critical to this systems-level analysis as we have identified many genes that are significantly differentially expressed when comparing the transcriptomes of primary breast tumors and metastases in breast cancer, but very few that are differentially expressed in independent datasets (unpublished observation). We found that fibulin genes fibulin-1, fibulin-2 and fibulin-5 were some of the most significantly differentially expressed genes in metastasis in breast cancer, meaning the magnitude of change in expression of theses genes between the primary breast tumor and metastasis was greater than the change in expression of $99 \%$ of the genes in the human genome.

Fibulin-1 is a proteoglycan component of the extracellular matrix. It binds hyaluronan through its amino terminus and with its carboxy terminus can bind the lectins versican and aggrecan (7). Fibulin-2 interacts with fibronectin (8) and, like Fibulin-1, can bind versican and aggregan (9). Fibulin-2 has also been shown to interact with fibrillin-1, a major component of 10-12 nanometer microfibrils that make up elastic tissues and are found in the extracellular matrix adjacent to basement membranes and elastic fibers (10). Fibulin-5, also known as DANCE, binds to elastin and is a required for elastogenesis, the development of elastic fibers $(11,12)$. Vascular smooth muscle cells cultured from fibulin- 5 deficient mice display heightened proliferative and migratory abilities after exposure to mitogens when compared to wildtype cells (13). Fibulin-5 over-expression in endothelial cells compromises their ability to undergo angiogenic sprouting (14). In mice, fibulin-5 is TGF-beta-inducible (15). Missense mutations in the fibulin- 5 gene cause a recessive form of cutis laxa, a 
hereditary connective tissue disorder that is characterized by loose skin (16). Thus, we have identified fibulin-5, a component that interacts with elastin, is required for the development of elastic fibers, and when mutated causes a genetic disorder that manifests itself as loosening of the skin, as one of the most differentially expressed and at significantly decreased levels in metastasis to the brain and soft tissues in breast cancer.

Fibulins have previously been implicated in cancer but this is the first report to demonstrate that these genes are differentially expressed in metastases to disparate organ sites in humans with breast cancer using transcriptome data generated from tissues isolated from patients diagnosed with breast cancer. In breast cancer, fibulin-1 has been shown to be over-expressed in primary tumor tissues when compared to normal breast tissues (17). A humoral response to fibulin-1 was detected in two patients whose dendritic cells could support a CD4+ T-cell response to fibulin-1 antigen presentation (18). Doxorubicin treatment of breast cancer cells in vitro leads to increased expression of fibulin-1 (19). Treatment of mice orthotopically injected with MDA-MB-361 cells in the presence of Matrigel, a reagent that contains fibulin-1, lead to in increase in doxorubicin resistance, and injection of monoclonal antibodies against fibulin-1 reversed this Matrigel-induced resistance (19). One study showed that expression of fibulin-2 is lost at the protein level in primary tumors of the breast (20). Fibulin- 2 is cleaved by the ADAMTS- 4 aggrecanase (21) and interaction of fibulin- 2 with the ADAMTS-12 metalloprotease blocks this cleavage (22). A study investigating fibulin- 5 showed that along with fibulin 3 it played an antagonistic role against tumor angiogenesis, or the growth of new blood vessels by a tumor in order to support itself by 
generating increased blood flow (23). In lung cancers, fibulin-5 suppresses invasive behavior by inhibiting the matrix metalloproteinase MMP-7 (24).

The requirement for these genes can be assessed in vitro by measuring the effect of over-expression on the proliferative capacity of breast cancer cell lines and immortalized tumor tissues from primary breast cancers, but of particular importance is the impact these genes may have on the metastatic process in breast cancer. Xenograft mouse models of metastatic breast cancer $(25,26)$ should be rigorously assessed to determine whether over-expression of fibulins 1, 2, and 5 in vivo, independently and in tandem exerts any effect on time to death, time to metastasis, as well as metastatic burden. 


\section{$\underline{\text { References }}$}

1. American Cancer Society. "Breast cancer facts \& figures 2017-2018." (2017)

2. Mehlen, Patrick, and Alain Puisieux. "Metastasis: a question of life or death." Nature Reviews Cancer 6.6 (2006): 449.

3. Weigelt, B., Peterse, J.L. and Van't Veer, L.J., 2005. Breast cancer metastasis: markers and models. Nature reviews cancer, 5(8), pp.591-602.

4. Iwamoto, T., Niikura, N., Ogiya, R., Yasojima, H., Watanabe, K.I., Kanbayashi, C., Tsuneizumi, M., Matsui, A., Fujisawa, T., Iwasa, T. and Shien, T., 2019. Distinct gene expression profiles between primary breast cancers and brain metastases from pairmatched samples. Scientific reports, 9(1), pp.1-8.

5. Sinn, B.V., Fu, C., Lau, R., Litton, J., Tsai, T.H., Murthy, R., Tam, A., Andreopoulou, E., Gong, Y., Murthy, R. and Gould, R., 2019. SET ER/PR: a robust 18-gene predictor for sensitivity to endocrine therapy for metastatic breast cancer. NPJ breast cancer, 5(1), pp.1-8.

6. Liang, Peng, and Arthur B. Pardee. "Analysing differential gene expression in cancer." Nature Reviews Cancer 3.11 (2003): 869.

7. Aspberg, A., Adam, S., Kostka, G., Timpl, R. and Heinegård, D., 1999. Fibulin-1 is a ligand for the C-type lectin domains of aggrecan and versican. Journal of Biological Chemistry, 274(29), pp.20444-20449.

8. Sasaki, T., Göhring, W., Pan, T.C., Chu, M.L. and Timpl, R., 1995. Binding of mouse and human fibulin-2 to extracellular matrix ligands. Journal of molecular biology, 254(5), pp. 892-899.

9. Olin, A.I., Mörgelin, M., Sasaki, T., Timpl, R., Heinegård, D. and Aspberg, A., 2001. The proteoglycans aggrecan and Versican form networks with fibulin-2 through their lectin domain binding. Journal of Biological Chemistry, 276(2), pp.1253-1261.

10. Reinhardt, D.P., Sasaki, T., Dzamba, B.J., Keene, D.R., Chu, M.L., Göhring, W., Timpl, R. and Sakai, L.Y., 1996. Fibrillin-1 and fibulin-2 interact and are colocalized in some tissues. Journal of Biological Chemistry, 271(32), pp.19489-19496.

11. Nakamura, T., Lozano, P.R., Ikeda, Y., Iwanaga, Y., Hinek, A., Minamisawa, S., Cheng, C.F., Kobuke, K., Dalton, N., Takada, Y. and Tashiro, K., 2002. Fibulin-5/DANCE is essential for elastogenesis in vivo. Nature, 415(6868), pp.171-175.

12. Yanagisawa, H., Davis, E.C., Starcher, B.C., Ouchi, T., Yanagisawa, M., Richardson, J.A. and Olson, E.N., 2002. Fibulin-5 is an elastin-binding protein essential for elastic fibre development in vivo. Nature, 415(6868), pp.168-171.

13. Spencer, J.A., Hacker, S.L., Davis, E.C., Mecham, R.P., Knutsen, R.H., Li, D.Y., Gerard, R.D., Richardson, J.A., Olson, E.N. and Yanagisawa, H., 2005. Altered vascular remodeling in fibulin-5-deficient mice reveals a role of fibulin-5 in smooth muscle cell proliferation and migration. Proceedings of the National Academy of Sciences, 102(8), pp.2946-2951. 
14. Albig, A.R. and Schiemann, W.P., 2004. Fibulin-5 antagonizes vascular endothelial growth factor (VEGF) signaling and angiogenic sprouting by endothelial cells. DNA and cell biology, 23(6), pp.367-379.

15. Schiemann, W.P., Blobe, G.C., Kalume, D.E., Pandey, A. and Lodish, H.F., 2002. Contextspecific Effects of Fibulin-5 (DANCE/EVEC) on Cell Proliferation, Motility, and Invasion FIBULIN-5 IS INDUCED BY TRANSFORMING GROWTH FACTOR- $\beta$ AND AFFECTS PROTEIN KINASE CASCADES. Journal of Biological Chemistry, 277(30), pp.27367-27377.

16. Loeys, B., Van Maldergem, L., Mortier, G., Coucke, P., Gerniers, S., Naeyaert, J.M. and De Paepe, A., 2002. Homozygosity for a missense mutation in fibulin-5 (FBLN5) results in a severe form of cutis laxa. Human molecular genetics, 11(18), pp.2113-2118.

17. Greene, L.M., Twal, W.O., Duffy, M.J., McDermott, E.W., Hill, A.D., O'Higgins, N.J., McCann, A.H., Dervan, P.A., Argraves, W.S. and Gallagher, W.M., 2003. Elevated expression and altered processing of fibulin-1 protein in human breast cancer. British journal of cancer, 88(6), pp.871-878.

18. Pupa, S.M., Argraves, S.W., Forti, S., Casalini, P., Berno, V., Agresti, R., Aiello, P., Invernizzi, A., Baldassari, P., Otwal, W. and Mortarini, R., 2004. Immunological and pathobiological roles of fibulin-1 in breast cancer. Oncogene, 23(12), pp.2153-2160.

19. Pupa, S.M., Giuffré, S., Castiglioni, F., Bertola, L., Cantú, M., Bongarzone, I., Baldassari, P., Mortarini, R., Argraves, W.S., Anichini, A. and Menard, S., 2007. Regulation of breast cancer response to chemotherapy by fibulin-1. Cancer research, 67(9), pp.4271-4277.

20. Yi, C.H., Smith, D.J., West, W.W. and Hollingsworth, M.A., 2007. Loss of fibulin-2 expression is associated with breast cancer progression. The American journal of pathology, $170(5)$, pp. 1535-1545.

21. Fontanil, T., Álvarez-Teijeiro, S., Villaronga, M.Á., Mohamedi, Y., Solares, L., MoncadaPazos, A., Vega, J.A., García-Suárez, O., Pérez-Basterrechea, M., García-Pedrero, J.M. and Obaya, A.J., 2017. Cleavage of Fibulin-2 by the aggrecanases ADAMTS-4 and ADAMTS-5 contributes to the tumorigenic potential of breast cancer cells. Oncotarget, 8(8), p.13716.

22. Fontanil, T., Rúa, S., Llamazares, M., Moncada-Pazos, A., Quirós, P.M., García-Suórez, O., Vega, J.A., Sasaki, T., Mohamedi, Y., Esteban, M.M. and Obaya, A.J., 2014. Interaction between the ADAMTS-12 metalloprotease and fibulin-2 induces tumor-suppressive effects in breast cancer cells. Oncotarget, 5(5), p.1253.

23. Albig, A.R., Neil, J.R. and Schiemann, W.P., 2006. Fibulins 3 and 5 antagonize tumor angiogenesis in vivo. Cancer research, 66(5), pp.2621-2629.

24. Yue, W., Sun, Q., Landreneau, R., Wu, C., Siegfried, J.M., Yu, J. and Zhang, L., 2009. Fibulin-5 suppresses lung cancer invasion by inhibiting matrix metalloproteinase-7 expression. Cancer research, 69(15), pp.6339-6346. 
25. Liu, H., Kato, Y., Erzinger, S.A., Kiriakova, G.M., Qian, Y., Palmieri, D., Steeg, P.S. and Price, J.E., 2012. The role of MMP-1 in breast cancer growth and metastasis to the brain in a xenograft model. BMC cancer, 12(1), p.583.

26. Bäuerle, T., Hilbig, H., Bartling, S., Kiessling, F., Kersten, A., Schmitt-Gräff, A., Kauczor, H.U., Delorme, S. and Berger, M.R., 2008. Bevacizumab inhibits breast cancer-induced osteolysis, surrounding soft tissue metastasis, and angiogenesis in rats as visualized by VCT and MRI. Neoplasia, 10(5), pp.511-520. 
Table 1

\begin{tabular}{|c|c|c|c|c|c|c|}
\hline Rank & Probe ID & p-value & $\mathbf{t}$ & B & Gene & Gene name \\
\hline \multicolumn{7}{|l|}{ GSE125989 (brain) } \\
\hline 122 & 202995_s_at & 3.27E-04 & -3.9531785 & 0.2338 & FBLN1 & fibulin 1 \\
\hline 129 & 203088_at & 3.67E-04 & -3.9140808 & 0.1312 & FBLN5 & fibulin 5 \\
\hline 165 & 203886_s_at & $6.92 \mathrm{E}-04$ & -3.6962067 & -0.4336 & FBLN2 & fibulin 2 \\
\hline \multicolumn{7}{|l|}{ GSE124648 } \\
\hline 56 & 203886_s_at & $5.22 \mathrm{E}-22$ & -11.0511736 & 39.37848 & FBLN2 & fibulin 2 \\
\hline 63 & 203088_at & $1.33 \mathrm{E}-21$ & -10.9105135 & 38.46274 & FBLN5 & fibulin 5 \\
\hline 65 & 202994_s_at & $1.44 \mathrm{E}-21$ & -10.8980269 & 38.38155 & FBLN1 & fibulin 1 \\
\hline
\end{tabular}

Table 1: Fibulins 1, 2 and 5 are differentially expressed in metastases to the brain and the soft tissues in patients with breast cancer.

The rank of differential expression out of 22277 total transcripts (GSE125989) or 22283 total transcripts (GSE124648), Affymetrix ID, p-value, t, a moderated t statistic, B, the log-odds of differential expression between the two groups, log-fold change of gene expression, the gene symbol and gene name are shown in the table above. 
FBLN1

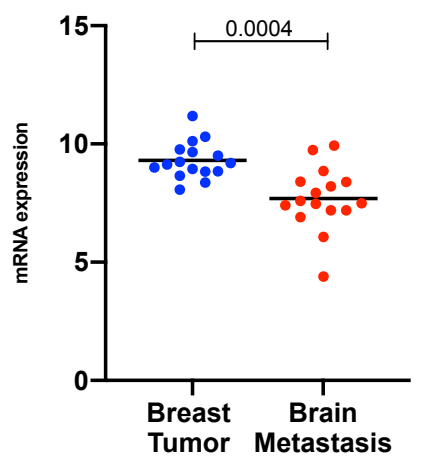

FBLN1

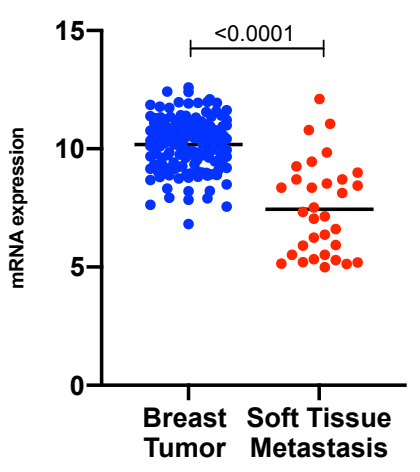

FBLN1

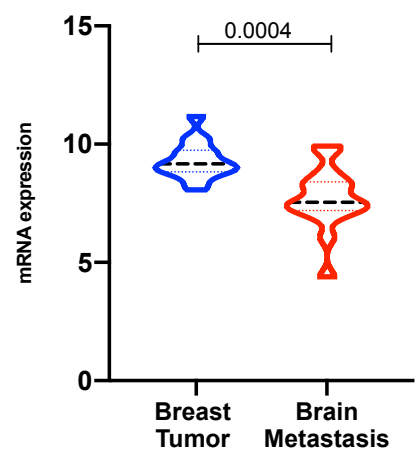

FBLN1

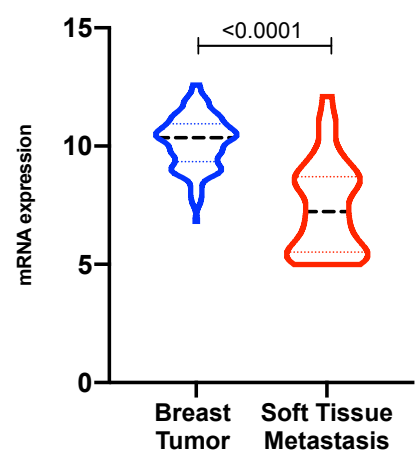

FBLN1

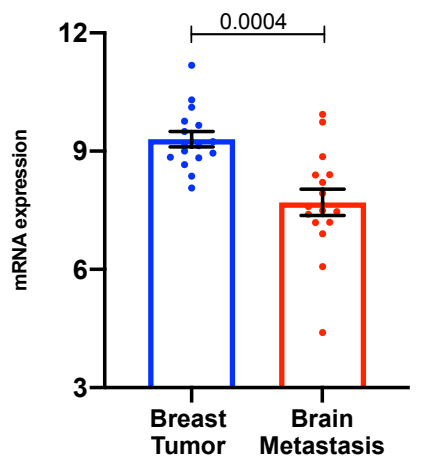

FBLN1

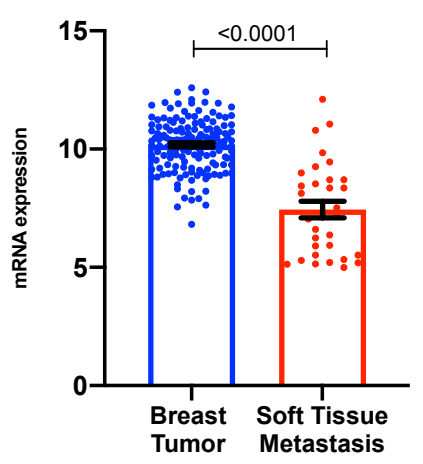

Figure 1: Fibulin 1 is expressed at significantly lower levels in metastases to the brain and the soft tissues when compared to primary tumors of the breast.

The expression of Fibulin 1 is graphically represented in blue (primary breast tumor) and in red (metastasis to the brain (top row) and metastasis to the soft tissues (bottom row)) with dots denoting the mRNA expression level in each individual patient and the black horizontal bar denoting the mean expression level (left), by a violin plot with the dotted black horizontal line denoting the median expression level (middle), and by a bar graph with the error bars denoting the standard error of the mean (right). The results of a statistical test evaluating the significance of difference between mRNA expression levels of Fibulin 1 in the primary and metastatic cancer tissues is shown as a p-value on each graph. 
FBLN2

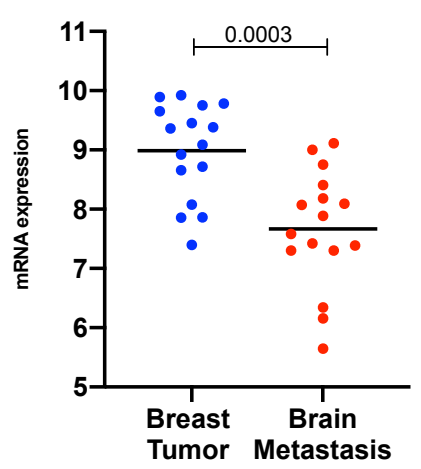

FBLN2

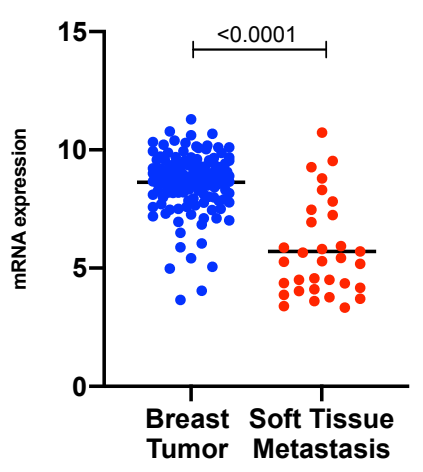

FBLN2

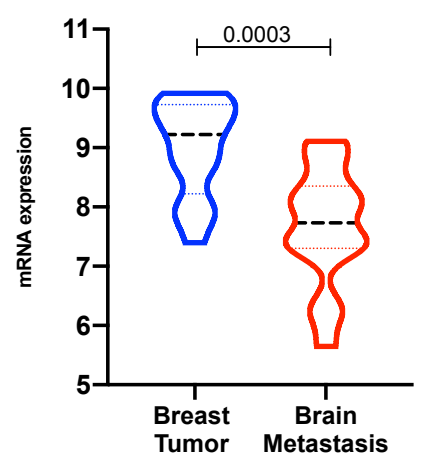

FBLN2

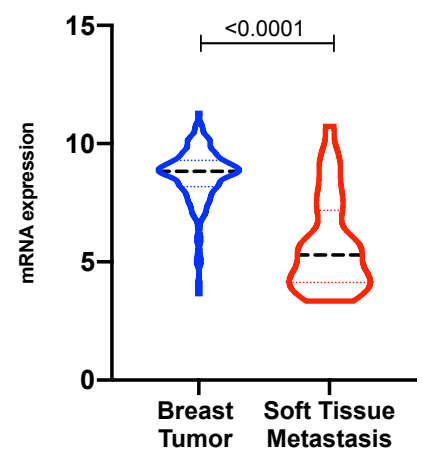

FBLN2

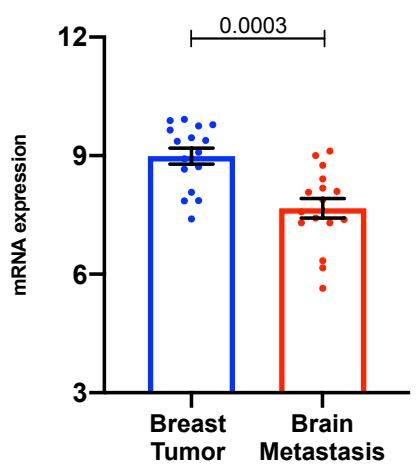

FBLN2

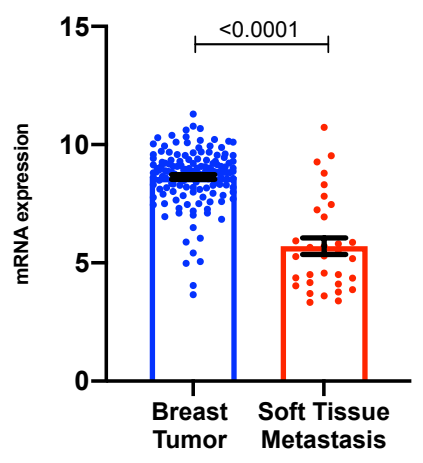

Figure 2: Fibulin 2 is expressed at significantly lower levels in metastases to the brain and the soft tissues when compared to primary tumors of the breast.

The expression of Fibulin 2 is graphically represented in blue (primary breast tumor) and in red (metastasis to the brain (top row) and metastasis to the soft tissues (bottom row)) with dots denoting the mRNA expression level in each individual patient and the black horizontal bar denoting the mean expression level (left), by a violin plot with the dotted black horizontal line denoting the median expression level (middle), and by a bar graph with the error bars denoting the standard error of the mean (right). The results of a statistical test evaluating the significance of difference between mRNA expression levels of Fibulin 2 in the primary and metastatic cancer tissues is shown as a p-value on each graph. 
FBLN5

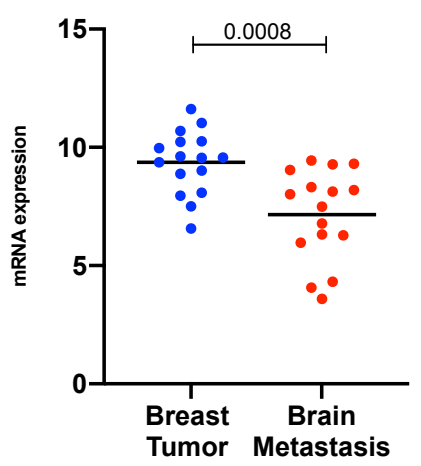

FBLN5

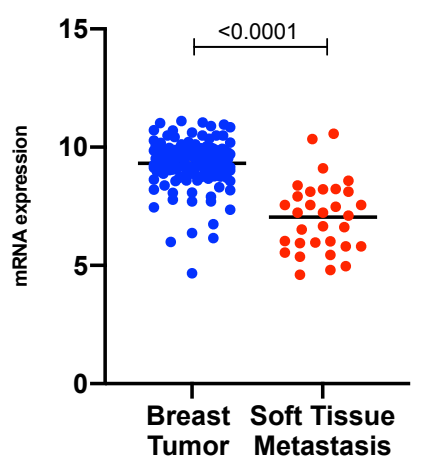

FBLN5

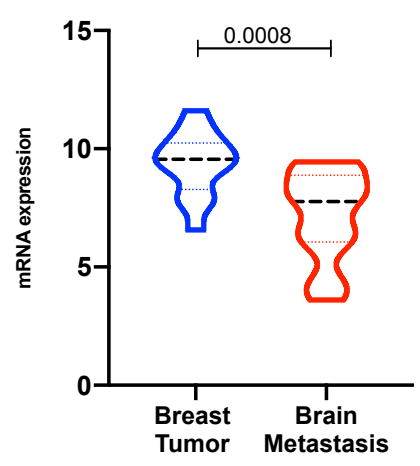

FBLN5

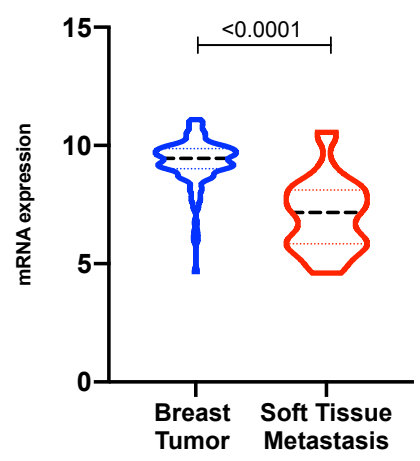

FBLN5

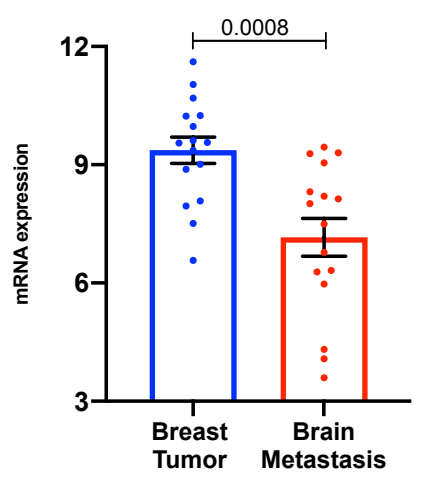

FBLN5

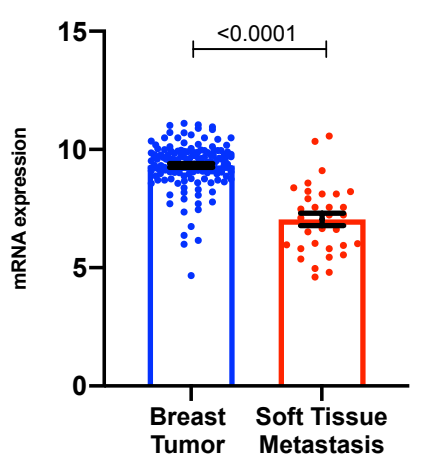

Figure 3: Fibulin 5 is expressed at significantly lower levels in metastases to the brain and the soft tissues when compared to primary tumors of the breast.

The expression of Fibulin 5 is graphically represented in blue (primary breast tumor) and in red (metastasis to the brain (top row) and metastasis to the soft tissues (bottom row)) with dots denoting the mRNA expression level in each individual patient and the black horizontal bar denoting the mean expression level (left), by a violin plot with the dotted black horizontal line denoting the median expression level (middle), and by a bar graph with the error bars denoting the standard error of the mean (right). The results of a statistical test evaluating the significance of difference between mRNA expression levels of Fibulin 5 in the primary and metastatic cancer tissues is shown as a p-value on each graph. 